Rosel Mieko Yamamoto Nomura'

APARECIDO NAKANO MARTINS ${ }^{2}$

Letícia Kamio Teshima ${ }^{2}$

SeIzo MIYADAHIRA ${ }^{1}$

MARCelo ZUGAiB ${ }^{3}$

\section{Movimentos respiratórios fetais em gestações com diabetes mellitus pré-gestacional}

\author{
Fetal breathing movements in pregnancies complicated by pregestational \\ diabetes mellitus
}

\section{Palavras-chaves \\ Diabetes mellitus Gravidez em diabéticas Feto/fisiologia \\ Ultra-sonografia Gravidez de alto risco Líquido amniótico \\ Respiração \\ Monitorização fetal \\ Keywords \\ Diabetes mellitus Pregnancy in diabetics \\ Fetus/physiology Ultrasonography Pregnancy, high-risk Amniotic fluid Respiration Fetal monitoring}

\title{
Resumo
}

OBJETIVO: analisar o padrão dos movimentos respiratórios fetais (MRF) em gestantes diabéticas no terceiro trimestre de gestação. MÉTODOS: foram avaliadas 16 gestantes com diabetes mellitus pré-gestacional e 16 gestantes normais (grupo controle), com os seguintes critérios de inclusão: gestação única entre a $36^{a}$ e a $40^{a}$ semana, ausência de outras doenças maternas e ausência de anomalias fetais. No perfil biofísico fetal (PBF), foram avaliados os parâmetros: freqüência cardíaca fetal, MRF, movimentos corpóreos fetais, tônus fetal e índice de líquido amniótico. Os MRF foram avaliados por 30 minutos, período em que o exame foi integralmente gravado em fita de vídeo VHS para posterior análise do número de episódios de MRF, do tempo de duração dos episódios e do índice de movimentos respiratórios fetais (IMR). O IMR foi calculado pela fórmula: (intervalo de tempo com MRF/tempo de observação) x 100. No início e no final do PBF foi dosada a glicemia capilar materna. Os resultados foram analisados pelo teste de Mann-Whitney $U$ e teste exato de Fisher, adotando-se nível de significância de 5\%. RESULTADOS: as glicemias demonstraram média significativamente superior nas diabéticas (1 13,3 $135,3 \mathrm{~g} / \mathrm{dL}$ ) em relação às gestantes normais $(78,2 \pm 14,8 \mathrm{~g} / \mathrm{dL}, \mathrm{p}<0,001)$. A média do índice de líquido amniótico mostrou-se maior no grupo das gestantes diabéticas $(15,5 \pm 6,4 \mathrm{~cm})$ quando comparado aos controles (10,6 $\pm 2,0 \mathrm{~cm} ; \mathrm{p}=0,01)$. A média do número de episódios de MRF foi superior nas diabéticas $(22,6 \pm 4,4)$ em relação aos controles (14,8 2 2,3; $p<0,0001)$. A média do IMR nas diabéticas $(54,6 \pm 14,8 \%)$ foi significativamente maior do que no grupo controle $(30,5 \pm 7,4 \% ; p<0,0001)$. CONCLUSÕES: os maiores valores glicêmicos podem estar associados a diferente padrão nos movimentos respiratórios de fetos de mães diabéticas. A utilização deste parâmetro do PBF, na prática clínica, deve ser considerada de forma criteriosa nas gestantes diabéticas.

\section{Abstract}

PURPOSE: to analyze the pattern of fetal breathing movements (FBM) in diabetic pregnant women in the third trimester of pregnancy. METHODS: sixteen pregestational diabetic and 16 nondiabetic (control group) pregnant subjects were included fulfilling the following criteria: singleton, between 36-40 weeks of gestation, absence of other maternal diseases and absence of fetal anomalies. The fetal biophysical profile (FBP) was performed to evaluate the following parameters: fetal heart rate, FBM, fetal body movements, fetal tone and amniotic fluid index. The FBM was evaluated for 30 minutes, period when the examination was integrally recorded in VHS video for posterior analysis of the number of FBM episodes, the duration of each episode and the fetal breathing movements index (BMI). The BMI was calculated by the formula: (interval of time with FBM/total time of observation) $\times 100$. At the beginning and in the end of the FBP maternal glucose levels were checked. The results were analyzed by the Mann-Whitney U-test and the Fisher exact test, adopting a level of significance of $5 \%$. RESULTS: the glucose levels demonstrated significantly superior average in the diabetic group (1 $13.3 \pm 35.3 \mathrm{~g} / \mathrm{dL}$ ) in relation to the normal group $(78.2 \pm 14.8 \mathrm{~g} / \mathrm{dL}, \mathrm{p}<0.001)$. The average of the amniotic fluid index was higher in the group of the diabetic cases $(15.5 \pm 6.4 \mathrm{~cm})$ when compared with controls $(10.6 \pm 2.0 \mathrm{~cm} ; \mathrm{p}=0.01)$. The average of the number of FBM episodes was superior in the diabetic ones $(22.6 \pm 4.4)$ in relation to controls $(14.8 \pm 2.3 ; p<0.0001)$. The average of the $\mathrm{BMI}$ in the diabetic patients $(54.6 \pm 14.8 \%)$ was significantly higher than that in the control group $(30.5 \pm 7.4 \%, p<0.0001)$. CONCLUSIONS: the elevated blood glucose levels can be associated with a different pattern in the FBM of diabetic mothers. The use of this parameter of the FBP, in the obstetric practice, must be considered with concern in diabetic pregnancies.

Correspondência:

Roseli Mieko Yamamoto Nomura Rua General Canavarro, 280 - Bairro Campestre CEP 09070-440 - Santo André/SP Fone: (11) 4991-2481 - Fax (11) 4221-8752 E-mail: roseli.nomura@terra.com.br
Clínica Obstétrica do Hospital das Clínicas da Faculdade de Medicina da Universidade de São Paulo - USP - São Paulo (SP), Brasil. Bolsa de iniciação científica concedida pela Fundação de Amparo à Pesquisa do Estado de São Paulo - Fapesp - ao aluno Aparecido Nakano Martins.

- Professor Colaborador Médico do Departamento de Obstetrícia e Ginecologia da Faculdade de Medicina da Universidade de São Paulo - USP - São Paulo (SP), Brasil.

${ }^{2}$ Acadêmico em Medicina da Faculdade de Medicina da Universidade de São Paulo - USP - São Paulo (SP), Brasil.

3 Professor Titular do Departamento de Obstetrícia e Ginecologia da Faculdade de Medicina da Universidade de São Paulo USP - São Paulo (SP), Brasil. 


\section{Introdução}

Os movimentos respiratórios fetais (MRF) ocorrem no desenvolvimento normal do produto conceptual, iniciam-se a partir da $11^{\text {a }}$ semana de gestação e são observados até o termo ${ }^{1}$. A presença de episódios de MRF constitui um dos parâmetros analisados no perfil biofísico fetal (PBF), juntamente com a observação da freqüência cardíaca fetal (FCF), dos movimentos corpóreos fetais (MCF), do tônus fetal e do volume de líquido amniótico (LA) ${ }^{2}$. Esse método, utilizado na propedêutica fetal das gestações de alto risco, avalia a vitalidade fetal, estimando a qualidade da oxigenação do sistema nervoso central, podendo, em situações patológicas, indicar a gravidade da hipoxemia e/ou acidemia fetais. Indiretamente, essa técnica também avalia a probabilidade de óbito fetal e perinatal, além do risco de comorbidades após seu nascimento ${ }^{3,4}$.

Alterações nos padrões dos MRF estão associadas a distúrbios fetais em seres humanos, sendo que sua ausência ou diminuição podem significar hipoxemia, hipoglicemia, parto pré-termo, infecção ou sofrimento fetal ${ }^{5,6}$. A avaliação dos MRF pelo PBF é método propedêutico de grande aplicação nas gestações complicadas pelo diabetes mellitus pré-gestacional, entretanto, os distúrbios glicêmicos podem interferir no padrão das atividades biofísicas fetais ${ }^{7,8}$.

Estudos realizados no terceiro trimestre da gestação demonstram que os MRF incidem em 30 a $40 \%$ do tempo total de observação ${ }^{9}$. São movimentos episódicos, muito provavelmente devido ao processo de maturação de grupos neuronais específicos e pela integridade de estruturas supramedulares do sistema nervoso central ${ }^{10,11}$

São descritos fatores que alteram o padrão dos MRF, entre eles a hiperglicemia materna, que se associa à maior ocorrência dos episódios. Estudos demonstram que, após refeições e infusões intravenosas de soluções de glicose, ocorre aumento da incidência de $\mathrm{MRF}^{12,13}$. A hipoglicemia materna, por sua vez, parece relacionar-se com a redução dos $M R F^{14,15}$.

A utilização do PBF na propedêutica fetal, em gestações complicadas pelo diabetes mellitus pré-gestacional, pode ter sua interpretação dificultada por fatores que possam influenciar nos padrões fisiológicos fetais, tal como o estado materno hiperglicêmico ${ }^{6,7}$. A investigação desses aspectos traz maior compreensão sobre a interpretação dos parâmetros biofísicos utilizados na prática obstétrica. O presente estudo tem o objetivo de analisar aspectos relativos ao padrão dos MRF em gestações complicadas pelo diabetes mellitus pré-gestacional.

\section{Métodos}

Este trabalho foi desenvolvido na Clínica Obstétrica do Hospital das Clínicas da Faculdade de Medicina da Universidade de São Paulo (HCFMUSP). A população estudada incluiu gestantes com diagnóstico de diabetes mellitus pré-gestacional, acompanhadas no ambulatório de pré-natal, no período compreendido entre outubro de 2004 e setembro de 2006. Foram avaliadas 16 pacientes com diagnóstico de diabetes mellitus e 16 pacientes normais, que constituíram o grupo controle.

Este estudo é do tipo prospectivo, caso-controle e transversal. O projeto de pesquisa e o termo de consentimento livre e esclarecido foram aprovados pela comissão de ética em pesquisa do HCFMUSP (Comissão de Ética de Análise de Projetos de Pesquisa - CAPPesq) sob o número 436/04.

Foram adotados os seguintes critérios de inclusão: diagnóstico de diabetes mellitus tipo 1 ou tipo 2 , prévio à gravidez; idade gestacional entre a $36^{\mathrm{a}}$ e a $40^{\mathrm{a}}$ semana; gestação única; ausência de outras doenças maternas associadas e ausência de anomalias fetais. As pacientes foram selecionadas quando compareciam para a avaliação da vitalidade fetal, que é conduta rotineiramente realizada no seguimento dessas pacientes. As que preenchiam os critérios de inclusão propostos foram convidadas a participar do estudo após serem esclarecidas sobre os objetivos da investigação, assinando o termo de consentimento livre e esclarecido (TCLE).

O grupo controle foi composto por gestantes normais, selecionadas entre as que compareceram para atendimento no pré-natal de baixo risco e que preencheram os mesmos critérios propostos para o grupo estudo, mas sem diagnóstico de diabetes mellitus. Estas gestantes foram acompanhadas até o final da gravidez e foram incluídas somente as que não desenvolveram intercorrência clínica ou obstétrica e que apresentaram resultado perinatal normal. As gestantes do grupo controle foram também esclarecidas previamente sobre a pesquisa e concordaram em participar assinando o respectivo TCLE.

O PBF foi realizado utilizando-se aparelho de ultra-sonografia da marca Toshiba ${ }^{\circledR}$ modelo SSA-220A e aparelho de cardiotocografia da marca Hewlett Packard $^{\circledR}$ modelo série 50A. A gravação do exame foi realizada por 30 minutos em fita de vídeo VHS, por meio de equipamento de gravação diretamente conectado ao equipamento de ultra-sonografia. A análise da FCF foi efetuada pela cardiotocografia, realizada com a paciente em posição sentada, com registro do traçado cardiotocográfico por, pelo menos, dez minutos. A cardiotocografia foi considerada normal quando na presença de, no mínimo, duas acelerações transitórias no período de 
observação máxima de 40 minutos. O PBF foi realizado com a paciente em posição de semi-Fowler, com o intuito de se evitar a hipotensão materna. Imediatamente antes e após a realização do PBF, foi mensurada a glicemia materna por meio da glicemia capilar.

Os parâmetros biofísicos avaliados pela ultra-sonografia incluíram: FCF, MRF, MCF, tônus fetal e volume de LA, observados durante o período de 30 minutos. Os MRF foram tomados como normais quando o feto apresentou, no mínimo, um episódio com duração mínima de 30 segundos, ao se visualizar o tórax fetal no plano sagital. Os MCF foram caracterizados como normais quando o feto demonstrou pelo menos um movimento amplo ou três movimentos lentos. O tônus fetal foi considerado normal na presença de MCF ou quando o feto revelou movimentos de abertura e fechamento das mãos no intervalo supracitado. $\mathrm{O}$ volume de LA foi apreciado por meio da medida do índice de ILA, conforme técnica proposta por Phelan et al. ${ }^{16}$. Nessa técnica, é mensurado o diâmetro vertical do maior bolsão de LA de cada quadrante do abdome materno e seu somatório em centímetros é denominado ILA. Seus valores foram classificados como normais quando foram superiores a $5,0 \mathrm{~cm}$.

Cada parâmetro do PBF recebeu a pontuação 2 quando qualificado como normal e 0 quando anormal. Pelo somatório, houve classificação do PBF em normal (8 ou 10), suspeito (6) ou alterado (4,2 e 0 ).

Para estudo específico dos MRF, foi utilizado o índice de movimentos respiratórios (IMR), que corresponde à porcentagem do tempo em que o feto apresentava esses movimentos. Este índice já foi descrito por Trudinger et al. ${ }^{17}$, em 1978, e utilizando o mesmo método, foi calculado da seguinte maneira: IMR = intervalo de tempo em que o feto apresentou MRF x 100/tempo total de observação.

Para avaliação precisa do IMR, a monitoração da parede torácica e abdominal fetal foi feita pela ultra- sonografia durante os 30 minutos de observação, e as imagens gravadas em fita de vídeo foram posteriormente analisadas para a quantificação do tempo de duração de cada episódio de MRF, realizada com o auxílio de um cronômetro.

As características do grupo estudo e do grupo controle estão apresentadas na Tabela 1. Não foram observadas diferenças significativas quanto à idade materna $(29,6 \pm 7,2$ anos para o grupo estudo e $28,8 \pm 6,3$ anos para o grupo controle). O número de gestações, a paridade e a idade gestacional no exame foram semelhantes entre os grupos. Não foram observadas diferenças significativas quanto à idade gestacional no exame.

Os parâmetros do $\mathrm{PBF}$ foram analisados descritivamente. Para as variáveis quantitativas, esta análise foi feita pelo cálculo de médias, medianas e desvios padrão. A comparação das médias nos grupos foi realizada utilizando-se o teste não paramétrico de Mann-Whitney U e a comparação entre as proporções foi avaliada pelo teste exato de Fisher. O nível de significância utilizado para os testes foi $5 \%$.

\section{Resultados}

No presente estudo, foram avaliadas 32 gestantes, das quais 16 apresentavam diabetes mellitus pré-gestacional, sendo nove pacientes com diabetes tipo 1 e sete com diabetes tipo 2, e 16 gestantes normais, que constituíram o grupo controle.

Quanto à análise da glicemia capilar realizada por ocasião da investigação, foi realizada média entre as medidas antes e após exame ultra-sonográfico, que foi significativamente maior nas diabéticas $(113,31 \pm 35,25 \mathrm{~g} / \mathrm{dL})$ em relação às gestantes normais $(78,22 \pm 14,83 \mathrm{~g} / \mathrm{dL}$, $\mathrm{p}<0,001)$, conforme Tabela 1 .

Em relação aos resultados perinatais (Tabela 1), um recém-nascido (7\%) do grupo em estudo apresentou índice de Apgar do primeiro minuto inferior

Tabela 1 - Características maternas e dados da gestação de pacientes normais e das portadoras de diabetes mellitus pré-gestacional.

\begin{tabular}{|c|c|c|c|c|c|}
\hline \multirow{2}{*}{$\begin{array}{l}\text { Parâmetro } \\
\text { Idade materna, anos, média (DP) }\end{array}$} & \multicolumn{2}{|c|}{$\begin{array}{c}\text { Grupo diabetes } \\
\text { pré-gestacional }(n=16)\end{array}$} & \multicolumn{2}{|c|}{$\begin{array}{l}\text { Grupo controle } \\
(n=16)\end{array}$} & \multirow{2}{*}{$\frac{p}{0,894}$} \\
\hline & 29,6 & $(7,2)$ & 28,9 & $(6,3)$ & \\
\hline Idade gestacional, semanas, média (DP) & 36,9 & $(0,9)$ & 37,1 & $(0,8)$ & 0,41 \\
\hline Paridade, média (DP) & 1,2 & $(1,0)$ & 0,6 & $(0,7)$ & 0,05 \\
\hline Número de abortos, média (DP) & 0,6 & $(0,9)$ & 0,1 & $(0,3)$ & 0,06 \\
\hline Peso do RN, gramas, média (DP) & 3672,1 & $(452,3)$ & 3229,4 & $(280,2)$ & 0,010 \\
\hline Apgar $1^{\circ} \min <7, \mathrm{n}(\%)$ & 1 & 6,3 & - & - & 0,953 \\
\hline Apgar $5^{\circ} \mathrm{min}<7, \mathrm{n}(\%)$ & 0 & - & - & - & - \\
\hline
\end{tabular}


a sete e nenhum no grupo controle $(0 \%, \mathrm{p}=0,953)$. Uma paciente diabética ainda está com a gestação em andamento.

A idade gestacional no nascimento foi significativamente $(\mathrm{p}<0,005)$ menor no grupo de gestantes com diabetes pré-gestacional quando comparado ao controle (Tabela 1).

$\mathrm{O}$ PBF apresentou-se normal $(\mathrm{PBF}=10)$ em todos os casos. Na Tabela 2, verifica-se que, apesar de o resultado final do PBF ser caracterizado como normal, a média do ILA mostrou valor significativamente maior no grupo das gestantes diabéticas $(15,5 \pm 6,4$ $\mathrm{cm})$ quando comparado aos controles $(10,6 \pm 2,0 \mathrm{~cm}$; $\mathrm{p}=0,01)$.

Quanto à análise dos MRF, a média do número de episódios foi significativamente superior nas diabéticas $(22,6 \pm 4,4)$ em relação aos controles $(14,8 \pm 2,3 ; \mathrm{p}<0,0001)$, conforme Figura 1. Não houve diferença na duração do maior e do menor episódio de MRF entre os grupos. $\mathrm{Na}$ Tabela 2 e na Figura 2, demonstra-se que a média do IMR nas diabéticas $(54,6 \pm 14,8 \%)$ demonstrou ser significativamente maior quando comparada ao grupo controle $(30,5 \pm 7,4 \% ; \mathrm{p}<0,0001)$.

As representações gráficas dos resultados do número de episódios de MRF e do IMR nos grupos estudados estão demonstradas nas Figuras 1 e 2 .

\section{Discussão}

O início dos MRF, durante o período de vida intra-uterino, indica a funcionalidade da inervação dos motoneurônios dos centros respiratórios e dos músculos por estes inervados. O desenvolvimento dos motoneurônios respiratórios ocorre durante o estágio fetal e completa-se no período pós-natal ${ }^{1,10,11}$.

A maturação do sistema nervoso central pode ser protelada ou comprometida por estados hiperglicêmicos, dessa forma levando a alteração nas atividades biofísicas fetais, tais como FCF, MRF e MCF, ou mascarando possíveis sinais de hipoxemia ${ }^{18}$. O próprio padrão de comportamento fetal, relacionado ou não ao diabetes, caracterizado pela maior ou menor atividade fetal, pode influenciar na análise dos parâmetros biofísicos fetais ${ }^{19}$.

Na presente pesquisa, os resultados obtidos na análise do PBF não demonstram diferenças significativas no índice final do exame, pois não apresentam diferenças nas pontuações entre os grupos. O PBF, por ser exame habitualmente utilizado na avaliação do bem-estar fetal,

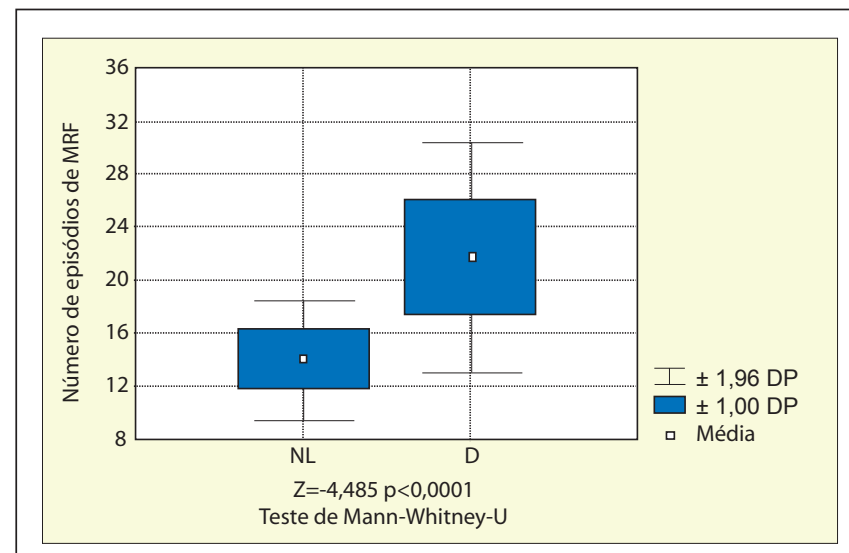

Figura 1 - Número de episódios de movimentos respiratórios fetais (MRF) em 30 minutos de observação, em gestantes diabéticas pré-gestacionais (D) comparadas ao grupo controle (NL).

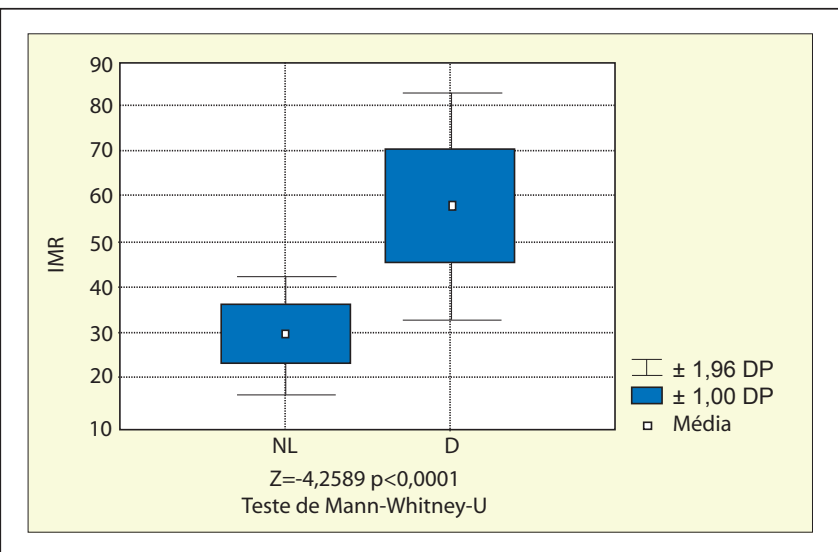

Figura 2 - Índice de movimentos respiratórios fetais (IMR) em 30 minutos de observação, em gestantes diabéticas pré-gestacionais (D) comparadas ao grupo controle (NL).

Tabela 2 - Características do perfil biofísico fetal (PBF) e avaliação do padrão dos movimentos respiratórios fetais (MRF).

\begin{tabular}{|c|c|c|c|c|c|}
\hline \multirow{2}{*}{$\begin{array}{l}\text { Parâmetro } \\
\text { PBF normal, n (\%) }\end{array}$} & \multicolumn{2}{|c|}{$\begin{array}{c}\text { Grupo diabetes } \\
\text { pré-gestacional }(n=16)\end{array}$} & \multicolumn{2}{|c|}{$\begin{array}{l}\text { Grupo controle } \\
(n=16)\end{array}$} & \multirow{2}{*}{$\frac{P}{-}$} \\
\hline & 16 & $100 \%$ & 16 & $100 \%$ & \\
\hline Índice de líquido amniótico, cm, média (DP) & 15,5 & $(6,4)$ & 10,6 & $(2,0)$ & 0,012 \\
\hline Episódio de MRF de maior duração, seg, média (DP) & 85,5 & $(27,4)$ & 73,0 & $(24,8)$ & 0,234 \\
\hline Episódio de MRF de menor duração, seg, média (DP) & 17,1 & $(7,1)$ & 19,8 & $(6,2)$ & 0,09 \\
\hline
\end{tabular}

IMR=índice de movimentos respiratórios. 
pode necessitar de diferente interpretação de parâmetros específicos nas gestantes diabéticas, nas quais a FCF basal, os episódios de variação da FCF, os MRF e o ILA podem estar associados aos valores de glicemia materna. Tanto os resultados falsos-negativos quanto os falsos-positivos são mais comuns em todas as modalidades da monitoração fetal em gestações complicadas pelo diabetes, quando comparadas a outras gestações de alto risco ${ }^{20}$.

Em fetos normais, a frequiência dos MRF apresenta correlação diretamente proporcional com o desenvolvimento pulmonar (débito cardíaco e fluxo sanguíneo para os órgãos vitais como coração, sistema nervoso central e placenta), contribuindo para homeostasia fetal; entretanto, não há informações em fetos de pacientes diabéticas ${ }^{1}$. A observação de maiores valores no IMR nas gestantes diabéticas em relação ao grupo controle, verificada no presente trabalho, assim como o maior número de episódios de MRF poderiam ser explicados pela hiperglicemia materna. Algumas hipóteses que procuram explicar a fisiopatologia desse mecanismo incluem a hipercapnia decorrente do metabolismo da glicose e a imaturidade relativa do sistema nervoso ${ }^{20,21}$. A hipercapnia fetal também é associada a maior incidência dos MRF com maior profundidade na fase inspiratória, resultado da estimulação de quimioreceptores centrais e periféricos ${ }^{1}$.

Contudo, outros mecanismos, além da hiperglicemia, podem estar envolvidos no aumento dos MRF nas gestantes diabéticas. Em estudo que analisa o PBF em gestantes diabéticas com controle glicêmico adequado, verifica-se maior incidência de $\mathrm{MRF}$, FCF e proporção de MRF nessas quando comparadas às gestantes normais; entretanto, verificam-se também menores taxas de MCF e menor número de acelerações da $\mathrm{FCF}^{8}$.

O aumento do volume de LA é alteração que se correlaciona às gestações complicadas pelo diabetes mellitus, sendo que já foi relatado que $15 \%$ dos casos de polidrâmnio ocorrem em pacientes diabéticas ${ }^{22}$. Uma teoria aceita é de que a hiperglicemia materna induza a hiperglicemia fetal e, conseqüentemente, a diurese osmótica, levando a aumento do líquido amniótico. Essa teoria é confirmada por estudos que demonstram, em gestantes diabéticas, aumento nos níveis de glicose no LA associado a valores mais elevados do ILA ${ }^{23}$.

Existem estudos que indicam relação entre polidrâmnio e macrossomia, assim como entre o aumento do ILA e maior peso do recém-nascido, tanto em gestantes diabéticas quanto em não-diabéticas $^{24}$. Além disso, estudos verificam relação linear entre o valor do ILA e o peso do recém-nascido em gestantes com diabetes mellitus com mau controle glicêmico ${ }^{22}$.

A gravidez em pacientes com diabetes mellitus está associada a maior risco de malformações fetais, complicações obstétricas e morbidade neonatal. São relatadas maiores taxas de prematuridade, parto cesárea, macrossomia e malformações fetais em gestantes complicadas pelo diabetes mellitus do tipo 1. A ocorrência de malformações congênitas e de macrossomia fetal tem correlação com os níveis da hemoglobina glicada durante a gestação, demonstrando a importância do controle glicêmico adequado para prevenção dessas alterações ${ }^{25}$. No presente estudo, recém-nascidos de gestantes diabéticas apresentaram maior peso no nascimento que o grupo controle, não sendo observado nenhum recém-nascido, no grupo controle, com peso superior a 4.000 gramas.

Apesar de o resultado final do PBF não apresentar diferença entre os grupos analisados, nas gestações com diabetes pré-gestacional verificou-se aumento do volume de LA, quando avaliado pelo ILA. O peso dos recém-nascidos também demonstrou ser superior nas gestações complicadas pelo diabetes quando comparado com as gestantes normais.

Pela análise do padrão dos MRF, o presente estudo confirma que os fetos de mães diabéticas apresentam movimentos respiratórios em proporção superior à observada nas gestantes normais, com maior número de episódios, o que pode estar relacionado aos maiores valores glicêmicos observados durante os exames ou alterações metabólicas decorrentes da hiperglicemia fetal. A utilização destes parâmetros do PBF, na prática clínica, deve ser considerada de forma criteriosa nas gestantes diabéticas.

\section{Agradecimento}

Agradecemos à Fundação de Amparo à Pesquisa do Estado de São Paulo -Fapesp - pela bolsa de iniciação científica concedida ao aluno Aparecido Nakano Martins para a elaboração do presente estudo. 
1. Abu-Shaweesh JM. Maturation of respiratory reflex responses in the fetus and neonate. Semin Neonatol. 2004; 9(3):169-80.

2. Manning FA. Dynamic ultrasound-based fetal assessment: the fetal biophysical profile score. Clin Obstet Gynecol. 1995; 38(1):26-44.

3. Manning FA. Fetal biophysical profile: a critical appraisal. Clin Obstet Gynecol. 2002; 45(4):975-85.

4. Nomura RMY, Francisco RPV, Maganha CA, Miyadahira S, Banduki Neto JD, Zugaib M. Vitalidade fetal em gestações complicadas com diabete melito pré-gestacional: um estudo longitudinal. Rev Bras Ginecol Obstet. 2002; 24(2): 1 13-20.

5. Vintzileos AM, Campbell WA, Ingardia CJ, Nochimson DJ. The fetal biophysical profile and its predictive value. Obstet Gynecol. 1983; 62(3):271-8

6. Honest H, Bachmann LM, Sengupta R, Gupta JK, Kleijnen J, Khan KS. Accuracy of absence of fetal breathing movements in predicting preterm birth: a systematic review. Ultrasound Obstet Gynecol. 2004; 24(1):94-100.

7. Landon $M B$, Gabbe SG. Fetal surveillance in the pregnancy complicated by diabetes mellitus. Clin Obstet Gynecol. 1991; 34(3):535-43.

8. Devoe LD, Youssef AA, Castillo RA, Croom CS. Fetal biophysical activities in third-trimester pregnancies complicated by diabetes mellitus. Am J Obstet Gynecol. 1994; 171 (2):298-303.

9. Patrick J, Campbell K, Carmichael L, Natale R, Richardson B. Patterns of human fetal breathing during the last 10 weeks of pregnancy. Obstet Gynecol. 1980; 56(1):24-30.

10. Greer JJ, Funk GD. Perinatal development of respiratory motoneurons. Respir Physiol Neurobiol. 2005; 149(1-3):43-61.

11. Greer JJ, Funk GD, Ballanyi K. Preparing for the first breath: prenatal maturation of respiratory neural control. J Physiol. 2006; 570(P+ 3):437-44.

12. Goodman JD. The effect of intravenous glucose on human fetal breathing measured by Doppler ultrasound. Br J Obstet Gynaecol. 1980; 87(12): 1080-3.

13. Bocking A, Adamson L, Cousin A, Campbell K, Carmichael L, Natale $R$, et al. Effects of intravenous glucose injections on human fetal breathing movements and gross fetal body movements at 38 to 40 weeks' gestational age. Am J Obstet Gynecol. 1982; 142(6 P+ 1):606-11.
14. Mirghani HM, Weerasinghe DS, Ezimokhai M, Smith JR. The effect of maternal fasting on the fetal biophysical profile. Int J Gynaecol Obstet. 2003; $81(1): 17-21$.

15. Mirghani HM, Weerasinghe SD, Smith JR, Ezimokhai M. The effect of intermittent maternal fasting on human fetal breathing movements. J Obstet Gynaecol. 2004; 24(6):635-7.

16. Phelan JP, Smith CV, Broussard P, Small M. Amniotic fluid volume assessment with the four-quadrant technique at 36-42 weeks' gestation. J Reprod Med. 1987; 32(7):540-2.

17. Trudinger BJ, Lewis PJ, Mangez J, O'Connor E. Fetal breathing movements in high risk pregnancy. Br J Obstet Gynaecol. 1978; 85(9):662-7.

18. Rudge MVC, Calderon IMP. A monitorização da hipóxia fetal nas gestações complicadas pelo diabete. Rev Bras Ginecol Obstet. 2005; 27(12):709-11.

19. Zisser H, Jovanovic L, Thorsell A, Kupperman A, Taylor L, Ospina $P$, et al. The fidgety fetus hypothesis: fetal activity is an additional variable in determining birth weight of offspring of women with diabetes. Diabetes Care. 2006; 29(1):63-7.

20. Walkinshaw SA. Pregnancy in women with pre-existing diabetes: management issues. Semin Fetal Neonatal Med. 2005; 10(4):307-15.

21. Florido J, Cortes E, Gutierrez M, Soto VM, Miranda MT, Navarrete L. Analysis of fetal breathing movements at 30-38 weeks of gestation. J Perinat Med. 2005; 33(1):38-41.

22. Vink JY, Poggi SH, Ghidini A, Spong CY. Amniotic fluid index and birth weight: is there a relationship in diabetics with poor glycemic control? Am J Obstet Gynecol. 2006; 195(3):848-50.

23. Dashe JS, Nathan L, Mclntire DD, Leveno KJ. Correlation between amniotic fluid glucose concentration and amniotic fluid volume in pregnancy complicated by diabetes. Am J Obstet Gynecol. 2000; 182(4):901-4.

24. Ott WJ. Reevaluation of the relationship between amniotic fluid volume and perinatal outcome. Am J Obstet Gynecol. 2005; 192(6): 1803-9.

25. Evers IM, Valk HW, Visser GH. Risk of complications of pregnancy in women with type 1 diabetes: nationwide prospective study in the Netherlands. BM. 2004; 328(7445):915. 